\title{
HSP90-targeted SN-38 Conjugate PEN-866
}

National Cancer Institute

\section{Source}

National Cancer Institute. HSP90-targeted SN-38 Conjugate PEN-866. NCI Thesaurus. Code C151953.

A miniature drug conjug ate composed of the irinotecan metabolite 7-ethyl-10-hydroxycamptothecin (SN-38) conjug ated, through a cleavable linker, to a ligand of chaperone protein heat shock protein 90 (Hsp90), with potential antineoplastic activity. Upon administration of HSP90-targeted SN-38 conjug ate PEN-866, the HSP90 ligand moiety targets HSP90, which allows the conjugate to penetrate, accumulate and be retained in the tumor cell. Once the linker is cleaved, the SN-38 moiety is released in a sustained manner. SN-38 then binds to and inhibits topoisomerase I by stabilizing the cleavable complex between topoisomerase I and DNA, which results in DNA breaks, inhibition of DNA replication and apoptosis. Compared to SN-38 alone, PEN-866 preferentially targets, accumulates and is retained in the tumor cells due to its binding to Hsp90, which results in increased concentrations of SN-38 at the tumor site. This allows sustained release of SN-38 and leads to increased and prolonged efficacy while reducing toxicity to normal, healthy tissues. Hsp90, a chaperone protein upregulated and activated in a variety of tumor cells compared to normal healthy tissue, regulates the folding, stability and degradation of many oncogenic signaling proteins. 